(Inflammatory Neuropathy Cause And Treatment-)Kriterien erfüllten, erhielten zunächst eine initiale Dosis von 2 g IVIG$\mathrm{C} / \mathrm{kg}$ Körpergewicht über einen Zeitraum von zwei bis maximal vier Tagen (Startdosis meist in zwei Infusionen) und danach eine Erhaltungsdosis in einer Infusion von $1 \mathrm{~g} / \mathrm{kg} \mathrm{KGW}(\mathrm{n}=59)$ oder Placebo $(\mathrm{n}=58)$ alle drei Wochen über einen Zeitraum von maximal 24 Wochen. Zeigte sich keine Veränderung (INCAT-Disability Score $<1$ ), hatten die Patienten in der Cross-over-Phase die Möglichkeit, auf den jeweils anderen Behandlungsarm zu wechseln. Die Extensionsphase diente dem Nachweis eines langfristigen Therapieerfolges. Beim primären Studienendpunkt (Therapieerfolg über sechs Monate) war Verum Placebo signifikant überlegen $(p=0,0002)$. Ebenso beim sekun- dären Endpunkt „Muskelkraft“: unter Immunglobulintherapie nahm die Griffstärke der dominanten $(\mathrm{p}=0,0008)$ sowie der nicht-dominanten Hand $(\mathrm{p}=0,005)$ im Placebovergleich deutlich zu. Die Langzeittherapie verbesserte zudem die gesundheitsbezogene Lebensqualität, wie die Auswertungen der Rotterdam Handicap Scale (RHS) und aller Bereiche des SF-36 (Short-Form-36) verdeutlichten, darunter insbesondere die körperliche Funktionsfähigkeit und Rollenfunktion, die soziale Funktionsfähigkeit sowie das psychische Wohlbefinden.

Dr. Yvette C. Zwick, freie Medizinjournalistin

Symposium "Aktuelles zu Immunopathien: Diagnostik und Therapie - wertvolle Tipps" im Rahmen des 85. DGN-Kongresses, Hamburg, 26.9.12. Veranstalter: Grifols Deutschland $\mathrm{GmbH}$

\title{
Oraler Immunmodulator reduziert Krankheitsprogression und Hirnatrophie
}

Studien mit Laquinimod zeigen unterschiedlich starke Wirkungen auf die Progression der Multiplen Sklerose (MS) und Hirnatrophie einerseits und die Reduktion der Schubraten andererseits. Eine Erklärung für diese Diskrepanz können die zentralen Zielzellen der LaquinimodWirkung sein.

„Der orale Immunmodulator Laquinimod ist sehr gut gehirngängig“, erklärte PD Dr. Martin Weber, Neurologische Klinik und Institut für Neuropathologie, Universität Göttingen. Neben dem Effekt auf das periphere Immunsystem mit reduzierter Sekretion proinflammatorischer Zytokine sei der zentrale Mechanismus der Substanz die Wirkung auf ZNS eigene Zellen wie Oligodendrozyten, Astrozyten und Mikroglia, die die Demyelinisierung und axonale Schädigung vermindert. Laquinimod reduziert die Aktivierung der Mikroglia und über die Blockade des NFkappaB-Signalwegs die Aktivierung von Astrozyten. Gleichzeitig verhindert der Immunmodulator läsionsunabhängige Gewebsschäden, die vorwiegend zur Hirnatrophie führen. „Diese Mechanismen erklären die gute Wirksamkeit auf die Behinderung und die Hirnatrophie bei im Vergleich nicht so beeindruckender Wirkung auf die Schubrate“, so Weber.
Die unterschiedliche Effektivität hatte sich in zwei Studien gezeigt: In der ALLEGRO-Studie [Comi G et al., NEJM 2012; 366: 1000 -9] reduzierte Laquinimod die Schubrate um moderate $23 \%$ gegenüber Placebo. „Deutlich stärker beeinflusst wurden Behinderung und Atrophie, die wahrscheinlich eng miteinander vergesellschaftet sind", berichtete Weber. Die Behinderungsprogression wurde früh und stark um $36 \%$ gesenkt, der Verlust an Hirnvolumen um 32,8\%. Eine Subanalyse, die mit MTR (whole brain magnetization transfer ratio) einen Marker für läsionsunabhängige Gewebsschäden untersuchte, zeigte, dass diese Schädigungen komplett verhindert werden konnten.

Die BRAVO-Studie [Vollmer T et al., ECTRIMS/ACTRIMS 2011; Abstract 148] reproduzierte die frühe und nachhaltige Wirkung auf die Behinderung mit Reduktion um 32,5\% und auf die Hirnatrophie mit Reduktion um 28,7\% - bei aber ebenfalls nur moderater Schubratenreduktion.

Michael Koczorek, freier Medizinjournalist

Satellitensymposium „Standpunkte zur Multiplen Sklerose", beim 85. DGN-Kongress,

Hamburg, 27.9.2012

Veranstalter: Teva Pharma

\section{Pflanzliche Schlafmittel}

Die seniorengerechte Auswahl von Arzneien ist auch bei Sedativa wichtig. Benzodiazepine sowie die Z-Substanzen können durch ihre muskelrelaxierende Wirkung zu Gangunsicherheit oder Stürzen führen. Weiterhin treten häufig paradoxe psychiatrische Reaktionen (Unruhe, Reizbarkeit, Halluzinationen) und geistige Beeinträchtigungen auf. Die oft eingesetzten älteren, sedierenden Antihistaminika können anticholinerge Nebenwirkungen verursachen, die wiederum zu Schwindel führen, Mundtrockenheit und Miktionsbeschwerden auslösen oder verstärken und sogar ein delirantes Syndrom auslösen können, erinnert das Unternehmen Bionorica SE. Bei der seniorengerechten Auswahl von Medikamenten kann die im Herbst 2010 eingeführte Priscus-Liste helfen, die 83 als für geriatrische Patienten potenziell ungeeignet geltende Wirkstoffe aufführt. Darunter befinden sich ältere Antihistaminika und Benzodiazepine. Alternativ schlägt die Priscus-Liste hoch dosierte Baldrianpräparate zur Behandlung bei Insomnie oder eine nicht-medikamentöse Therapie der Schlafstörungen vor. Pflanzliche Arzneimittel wie Allunapret ${ }^{\circledR}$ seien eine Alternative $\mathrm{zu}$ vielen chemisch-synthetischen Schlafmitteln. Bei Ein- und Durchschlafstörungen sei die Wirksamkeit des Arzneimittels, das eine Kombination methanolischer Trockenextrakte aus Baldrian und Hopfen enthält, in klinischen Studien belegt. Die adenosinerge Wirkung von Baldrian erhöhe die Schlafbereitschaft und werde durch die melatonerge Wirkung des Hopfens ergänzt, die den natürlichen Schlaf-Wach-Rhythmus unterstütze.

Nach Informationen von Bionorica

\section{Alzheimer-Demenz}

Seit Mitte Dezember 2012 ist Memantine Merz $^{\circledast}$ verfügbar. Das Axura ${ }^{\oplus}$-identische Generikum zur Behandlung moderater bis schwerer Alzheimer-Demenz wird in den gleichen Dosierungen und Packungsgrößen angeboten wie das Original Axura ${ }^{\circledast}$. Auch die Galenik ist identisch. Farbe und Form der Tabletten ändern sich nicht.

Nach Informationen von Merz Pharmaceuticals 